doi: $10.15407 /$ ujpe62.01.0046

P.J. BAYMATOV, B.T. ABDULAZIZOV

Namangan State University

(316, Uychi Str., Namangan 716019, Republic of Uzbekistan; e-mail: baymatov1958@mail.ru)

\title{
CONCENTRATION DEPENDENCES OF THE ELECTRON EFFECTIVE MASS, FERMI ENERGY, AND FILLING OF SUBBANDS IN DOPED InAs/AlSb QUANTUM WELLS
}

\begin{abstract}
Results of researches concerning the properties of the two-dimensional (2D) degenerate electron gas in a single quantum well on the basis of the InAs/AlSb heterostructure are reported. The non-parabolic character of the InAs and AlSb conduction bands is described by a simplified Kane model. The dispersion curves for first three subbands are calculated, as well as the dependences of the Fermi energy, subband filling, and effective mass of electrons at the Fermi level on the total $2 D$ electron concentration. The obtained results are in good agreement with experimental data.

Keywords: quantum well, Kane model, subband dispersion, subband filling, two-dimensional electron gas, effective mass, cyclotron mass.
\end{abstract}

\section{Introduction}

Heterostructures on the basis of InAs and InSb semiconductors have been intensively studied recently [1-3]. Those semiconductors are characterized by a strong nonparabolicity of their conduction bands. This phenomenon and the character of the electron spin splitting in quantum wells (QWs) are studied with the use of the cyclotron resonance method [4-7]. However, only undoped heterostructures with two-dimensional (2D) electron concentrations up to $10^{12} \mathrm{~cm}^{-2}$ were examined in the cited works.

In the case of a non-parabolic conduction band, the electron concentration growth is accompanied by an increase of the effective mass of an electron at the Fermi level. In work [8], an InAs/AlSb heterostructure with a single QW was considered, in which the QW width was $L=15 \mathrm{~nm}$. The $2 \mathrm{D}$ electron concentration $n_{s}$ was varied from $2.7 \times 10^{11}$ to $8 \times 10^{12} \mathrm{~cm}^{-2}$, and an almost two-fold increase of the effective mass of an electron was revealed at that. The total concentration of the $2 \mathrm{D}$ electron gas, $n_{s}$, was measured, by using the Hall effect. By analyzing Shubnikovde Haas $(\mathrm{SdH})$ oscillations, the electron concentrations in three first size-quantized subbands $\left(n_{s}^{(1)}, n_{s}^{(2)}\right.$, $\left.n_{s}^{(3)}\right)$ were determined. However, the comparison of

(C) P.J. BAYMATOV, B.T. ABDULAZIZOV, 2017 the concentration dependence of the effective mass $m_{c}\left(n_{s}\right)$ with experimental data was performed only for the first subband. As a result, the calculated mass values coincided with the experimental ones only for the QW width $L=20.5 \mathrm{~nm}$. The experimental QW width was equal to $L=15 \mathrm{~nm}$.

The results of such researches play an important role for understanding the nature of the $2 \mathrm{D}$ electron gas: the structure of subbands, subband electron statistics, and so on. The aim of this work is to calculate the subband dispersion curves making allowance for the non-parabolic character of the conduction band and considering the dependence of the Fermi energy, subband concentrations, and effective mass of an electron at the Fermi level on the total $2 \mathrm{D}$ electron concentration $n_{s}$ in a single QW with the width $L=15 \mathrm{~nm}$ created on the basis of the InAs/AlAs heterostructure.

\section{Subband Dispersion}

Let us consider a single QW of the width $L$ (region $A$ ), which is confined between potential barriers of the height $V$ (region $B$ ). The energy is reckoned from the bottom of the conduction band in the InAs bulk. In this work, we neglect the deformation effects, which result from the mismatch between the InAs and AlSb lattice constants.

ISSN 2071-0194. Ukr. J. Phys. 2017. Vol. 62, No. 1 
In the effective mass approximation, the solution of the three-dimensional Schrödinger equation is tried in the form $\psi=e^{i\left(k_{x} x+k_{y} y\right)} \phi(z)$. Then the following one-dimensional equations can be written for regions $A$ and $B$ :

$\frac{\partial^{2} \phi_{A}(z)}{\partial z^{2}}+q^{2} \phi_{A}(z)=0, \quad q=\sqrt{\frac{2 m_{A}}{\hbar^{2}} E-k^{2}}$,

$\frac{\partial^{2} \phi_{B}(z)}{\partial z^{2}}-\chi^{2} \phi_{B}(z)=0, \quad \chi=\sqrt{\frac{2 m_{B}}{\hbar^{2}}(V-E)+k^{2}}$.

Here, $k^{2}=k_{x}^{2}+k_{y}^{2}$, and $m_{A, B}$ are the energydependent electron effective masses in materials $A$ and $B$. By solving Eqs. (1) and (2) and using the boundary conditions

$\phi_{A}(0)=\phi_{B}(0)$,

$\left.\frac{1}{m_{A}} \frac{d \phi_{A}(z)}{d z}\right|_{z=0}=\left.\frac{1}{m_{B}} \frac{d \phi_{B}(z)}{d z}\right|_{z=0}$,

$\phi_{A}(L)=\phi_{B}(L)$,

$\left.\frac{1}{m_{A}} \frac{d \phi_{A}(z)}{d z}\right|_{z=L}=\left.\frac{1}{m_{B}} \frac{d \phi_{B}(z)}{d z}\right|_{z=L}$,

we obtain the formula for the dispersion $E=E_{n}(k)$ :

$$
\begin{aligned}
& E=E_{\|}+E_{0}[\pi n- \\
& \left.-2 \operatorname{Arcsin}\left(\sqrt{\frac{\gamma^{2}\left(E-E_{\|}\right)}{\gamma(\gamma-1) E+\gamma V+\left(1-\gamma^{2}\right) E_{\|}}}\right)\right]^{2} .
\end{aligned}
$$

Here, $E_{\|}=\hbar^{2} k^{2} / 2 m_{A}, E_{0}=\hbar^{2} / 2 m_{A} L^{2}$, and $\gamma=$ $=m_{B} / m_{A}$. The non-parabolic character of the conduction bands in InAs and AlSb is taken into account, by using the simplified formula

$m_{A}=m_{A}(0)\left(1+\alpha_{A} E\right)$,

$m_{B}=m_{B}(0)\left(1+\alpha_{B}(E-V)\right)$,

where $m_{A, B}(0)$ are the effective masses of electrons at the bottom of the conduction bands in regions $A$ and $B$, and $\alpha_{A, B}$ are the parameters of band nonparabolicity in those regions.

Equation (5) is inconvenient for the description of the electron statistics, because it cannot be solved for

ISSN 2071-0194. Ukr. J. Phys. 2017. Vol. 62, No. 1 the variable $E$ or $k$. In the approximation of infinitely high wall, $V \rightarrow \infty$, this equation acquires a simple form: $E=E_{\|}+E_{0} \pi^{2} n^{2}$. This approximation, however, overestimates the energy values in comparison with Eq. (5) (see Fig. 1).

Let us assume that, at $k=0$ and for the given value of $n$, the numerical solution of Eq. (5) equals $E_{n}$. Then it is convenient to express the right-hand side of Eq. (5) in the form $E_{n}\left(1+\alpha_{A} E_{n}\right)$. If this quantity is assumed to be independent of $k$, Eq. (5) brings about the following approximation for $E(n, k)$ :

$E\left(1+\alpha_{A} E\right) \approx \frac{\hbar^{2} k^{2}}{2 m_{A}(0)}+E_{n}\left(1+\alpha_{A} E_{n}\right)$,

where $E_{n}$ is the bottom energy for the $n$-th subband. Its value for every $n=1,2,3, \ldots$ can be determined, first, numerically from Eq. (5) at $k=0$ and, afterward, by substituting the result into Eq. (8) for further applications. Then the bottom energy values obtained for every subband from Eq. (5) and from approximation (8) coincide with each other.

For the InAs/AlSb structure with the QW width $L=15 \mathrm{~nm}$, we obtain from Eq. (5) that $E_{1}=$ $=0.0445 \mathrm{eV}, E_{2}=0.154 \mathrm{eV}$, and $E_{3}=0.295 \mathrm{eV}$. The values of band parameters for InAs and AlSb - the energy gap width $E_{g}$, effective mass $m(0)$, nonparabolicity parameter $\alpha$, and conduction band jump $V-$ which were used at calculations, are quoted in Table.

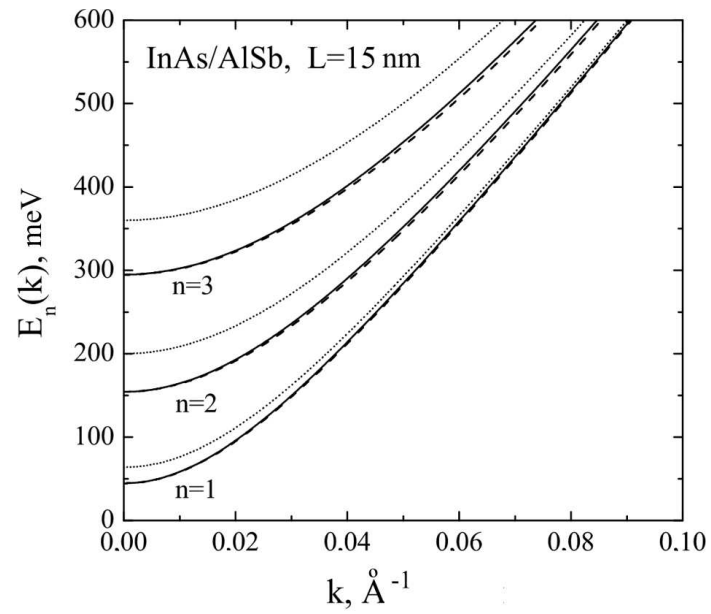

Fig. 1. Dispersion curves $E_{n}(k)$ for three first subbands $(n=1,2,3)$ in the InAs/AlSb quantum well with the width $L=15 \mathrm{~nm}$ : according to Eq. (5) (solid curves), according to approximation (8) (dashed curves), and at $V=\infty$ (dotted curves) 


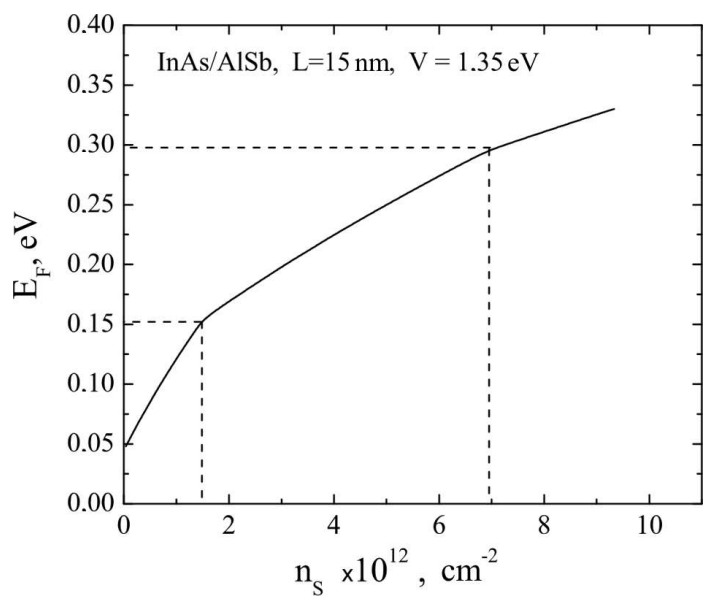

Fig. 2. Fermi energy dependence on the two-dimensional electron concentration in the InAs/AlSb quantum well with $L=15 \mathrm{~nm}$ and $V=1.35 \mathrm{eV}$

Band parameters for InAs and AlSb at 4.2 K

\begin{tabular}{|l|l|l|}
\hline & $\operatorname{InAs}(\mathrm{A})$ & $\mathrm{AlSb}(\mathrm{B})$ \\
\hline$E_{g}, \mathrm{eV}$ & 0.42 & 2.37 \\
$m(0)$ & 0.023 & 0.11 \\
$\alpha, \mathrm{eV}^{-1}$ & 2.27 & 0.334 \\
$V(z), \mathrm{eV}$ & 0 & 1.35 \\
\hline
\end{tabular}

The calculated dispersion curves $E_{n}(k)$ obtained according to Eq. (5) and approximation (8) are compared in Fig. 1. It is evident that approximation (8) is quite exact. It is convenient to be used, while studying the electron statistics and the kinetic, optical, or other parameters of the 2D electron gas. A shortcoming of approximation (8) consists in that $E_{n}$ depends on the structure parameters $L, V, m_{A, B}(0)$, and $\alpha_{A, B}$. Therefore, every time when those parameters change, the value of $E_{n}$ has to be recalculated numerically from Eq. (5) at $k=0$.

\section{Degenerate 2D Electron}

\section{Gas: Fermi Energy, Subband Filling}

The equation for the electron concentration can be written in the form

$n_{s}=N_{0} \sum_{n=1} \int_{E_{n}}^{E_{\mathrm{F}}} d\left(\frac{\hbar^{2} k^{2}}{2 m_{A}(0)}\right)$, where

$N_{0}=\frac{m_{A}(0)}{\pi \hbar^{2}}=\frac{m_{A}(0)}{m_{0}} \frac{0.413 \times 10^{15}}{\mathrm{eV} \mathrm{cm}^{2}}$.

Making allowance for approximation (8), we have

$n_{s}=\sum_{n=1} n_{s}^{(n)}$.

Here,

$n_{s}^{(n)}=N_{0}\left[E_{\mathrm{F}}+\alpha_{A} E_{\mathrm{F}}^{2}-\left(E_{n}+\alpha_{A} E_{n}^{2}\right)\right] \theta\left(E_{\mathrm{F}}-E_{n}\right)$

is the electron concentration in the $n$-th subband. In Eq. (10), the terms under the sum sign must be positive. Negative terms are excluded by the Heaviside function. Equation (10) establishes a relation between the Fermi energy $E_{\mathrm{F}}$ and the total 2D concentration of electrons $n_{s}$. It also determines the electron concentrations in separate subbands, $n_{s}^{(n)}$, for the given $n_{s}$.

Equation (10) makes it possible to evaluate the critical concentration $n_{c 1}$, at which the Fermi level reaches the bottom of the second subband, i.e. $E_{\mathrm{F}}=$ $=E_{2}$. In the InAs $/ \mathrm{AlSb}$ structure with the QW width $L=15 \mathrm{~nm}$, it equals

$n_{c 1}=N_{0}\left(E_{2}-E_{1}\right)\left[1+\alpha_{A}\left(E_{2}+E_{1}\right)\right]=$

$=1.5110^{12} \mathrm{~cm}^{-2}$.

According to the estimation of work [7], $n_{c 1} \approx 1.8 \times$ $\times 10^{12} \mathrm{~cm}^{-2}$, whereas the experimental value equals $1.2 \times 10^{12} \mathrm{~cm}^{-2}$, which is rather close to our one. Analogously, it is possible to evaluate the critical concentration $n_{c 2}$, at which the Fermi level reaches the bottom of the third subband, i.e. $E_{\mathrm{F}}=E_{3}$. It equals $n_{c 2} \approx 6.87 \times 10^{12} \mathrm{~cm}^{-2}$.

The dependence of the Fermi energy on the total electron concentration $E_{\mathrm{F}}\left(n_{s}\right)$ described by formula (10) is plotted in Fig. 2. One can see that the dependence $E_{\mathrm{F}}\left(n_{s}\right)$ includes cusps, at which the Fermi energy growth is slowed down. Those cusps reflect drastic changes (jumps) in the density of states at the critical points $n_{s}=n_{c 1}, E_{\mathrm{F}}=E_{2}, n_{s}=n_{c 2}$, and $E_{\mathrm{F}}=E_{3}$. They take place at the critical concentrations $n_{c 1}, n_{c 2}, \ldots$, when the Fermi level crosses the bottom of the next subband.

The dependences of the electron concentrations in the subbands on the total concentration, $n_{s}^{(n)}\left(n_{s}\right)$,

ISSN 2071-0194. Ukr. J. Phys. 2017. Vol. 62, No. 1 
are depicted in Fig. 3. These dependences can be obtained as follows. According to formula (10), the subband concentrations depend on the Fermi energy: $n_{s}^{(1)}\left(E_{\mathrm{F}}\right), n_{s}^{(2)}\left(E_{\mathrm{F}}\right)$, and $n_{s}^{(3)}\left(E_{\mathrm{F}}\right)$. Assigning various values to $E_{\mathrm{F}}$ (for example, from an interval of $0-0.4 \mathrm{eV}$ ), we can find the concentration in every subband, and their sum gives the total concentration $n_{s}$. So, we can plot graphically the dependences $n_{s}^{(n)}\left(n_{s}\right)$.

As the total concentration grows, the first subband becomes filled first. Its concentration increases linearly until the filling of the second subband begins. When the filling of the next subband begins, the growth in the dependences for the lower subbands becomes slower, and we obtain cusps. In the calculated dependences $n_{s}^{(n)}\left(n_{s}\right)$, those cusps are observed at the critical points: $n_{s}=n_{c 1}, E_{\mathrm{F}}=E_{2}$ and $n_{s}=n_{c 2}$, $E_{\mathrm{F}}=E_{3}$, being a result of a jump-like growth in the density of states.

Figure 3 also exhibits the experimental dependences of the electron concentrations in the subbands $n_{s}^{(n)}(\mathrm{SdH})$ on the total concentration $n_{s}$ (Hall) [8].

\section{Concentration Dependence of the Cyclotron Mass}

Similarly to the case of $3 \mathrm{D}$ systems $[9,10]$, let us establish a relation between the effective mass and the total $2 \mathrm{D}$ concentration of electrons, $m_{c}\left(n_{s}\right)$. In approximation (8), the effective mass at the Fermi level, $m_{c}\left(E_{\mathrm{F}}\right)$, does not depend on the subband number. Even at more exact calculations on the basis of Eq. (5), the effective masses in various subbands differ insignificantly. This fact is also confirmed by numerical calculations in work [8]. In approximation (8), the effective mass at the Fermi level equals

$m_{c}\left(E_{\mathrm{F}}\right)=m(0)\left(1+2 \alpha E_{\mathrm{F}}\right)$.

The plot of the dependence $m_{c}\left(n_{s}\right)$ is shown in Fig. 4. This dependence can be obtained by excluding $E_{\mathrm{F}}$ from Eqs. (10) and (12). Figure 4 also exhibits the experimental values of the effective (cyclotron) mass $m_{c}$ at the Fermi level measured at various total 2D electron concentrations [8]. The cusps in the dependence $m_{c}\left(n_{s}\right)$ are observed at the critical points $n_{s}=n_{c 1}, E_{\mathrm{F}}=E_{2}$, and $n_{s}=n_{c 2}$, $E_{\mathrm{F}}=E_{3}$.

From Fig. 4, one can see that the dependence $m_{c}\left(n_{s}\right)$ obtained in this work differs from the depen-

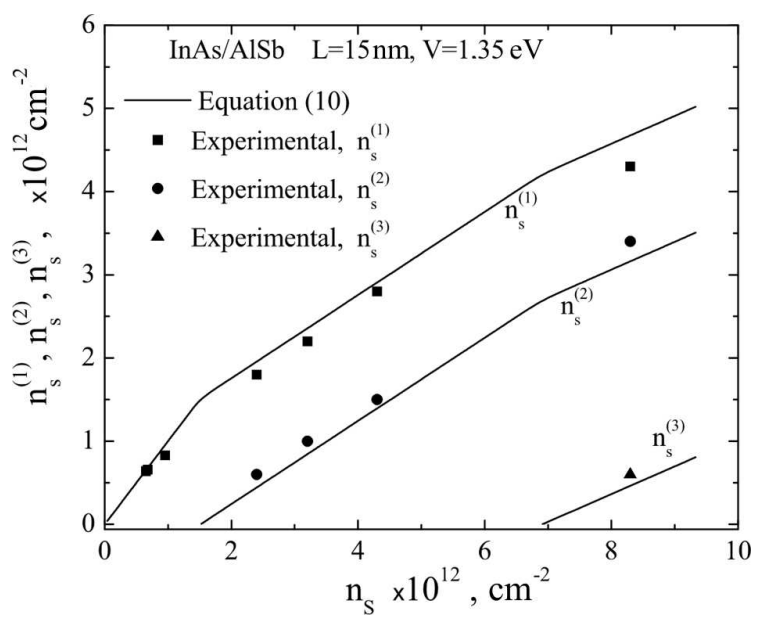

Fig. 3. Dependences of the electron concentrations in three first subbands $-n_{s}^{(1)}, n_{s}^{(2)}$, and $n_{s}^{(3)}-$ on the total twodimensional concentration in the InAs/AlSb quantum well with $L=15 \mathrm{~nm}$ and $V=1.35 \mathrm{eV}$, and their comparison with experimental data [8]

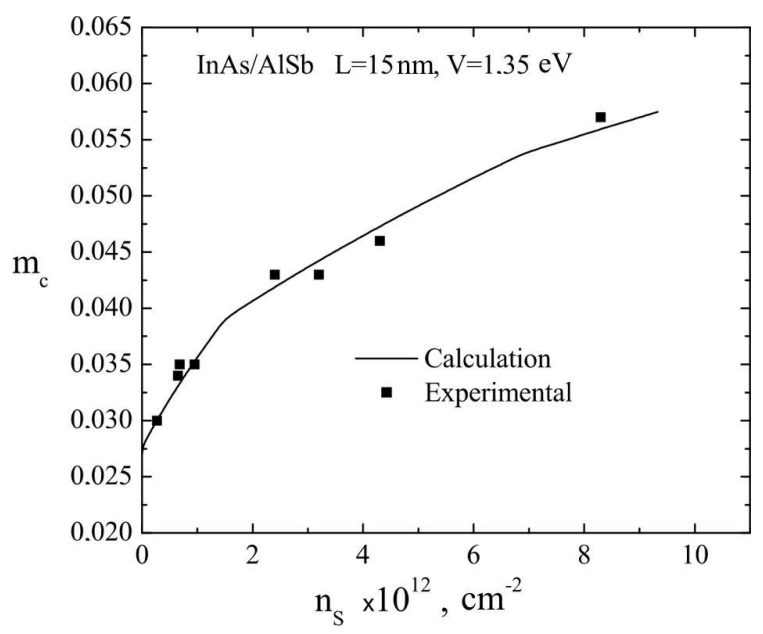

Fig. 4. Dependence of the effective mass on the total twodimensional concentration in the InAs/AlSb quantum well with $L=15 \mathrm{~nm}$ and $V=1.35 \mathrm{eV}$, and its comparison with experimental data [8]

dence given in work [8]. The total concentration $n_{s}$ is reckoned along the abscissa axis. The dependences $m_{c}\left(n_{s}\right)$ in Fig. 4 and $E_{\mathrm{F}}\left(n_{s}\right)$ in Fig. 2 are similar, because $m_{c}\left(E_{\mathrm{F}}\right)$ is proportional to $E_{\mathrm{F}}$. Hence, the nonparabolic dispersion law in the conduction band results in a monotonic growth of the effective mass with the concentration $n_{s}$. The slowdown of the growth rate at critical points is associated with a jump-like increase in the density of states. 


\section{Conclusions}

By experimentally varying the concentration of the $2 \mathrm{D}$ electron gas in a wide interval of $10^{11}-10^{13} \mathrm{~cm}^{-2}$, we can study the structures of emerged subbands, their relative filling, and the concentration dependence of the carrier effective mass. In this work, a useful approximation for the subband dispersion and simple relations for the calculation of the electron statistics, which satisfactorily describe experimental results [8], are obtained in the case of the degenerate electron gas in the InAs/AlSb quantum well heterostructure. The approximation is also useful for the calculation of the kinetic, optical, and magnetic properties of the 2D electron gas. Furthermore, the algorithms described above can be applied to other heterostructures with QWs on the basis of semiconductors of the $\mathrm{A}_{3} \mathrm{~B}_{5}$ group.

The work was carried out in the framework of the project of the State Scientific and Engineering Program (grant F2-OT-O-15494).

1. Yu.B. Vasilyev, F. Gouider, G. Nachtwei, P.D. Buckle. Cyclotron resonance in heterostructures with $\mathrm{InSb} / \mathrm{AlInSb}$ quantum wells. Fiz. Tekh. Poluprovodn. 44, 1559 (2010) (in Russian).

2. K.E. Spirin, K.P. Kalinin, S.S. Krishtopenko, K.V. Maremyanin, V.I. Gavrilenko, Yu.G. Sadofyev. Peculiarities of persistent photoconductivity in InAs/AlSb double quantum well heterostructures with tunnel transparent separation barrier. Fiz. Tekh. Poluprovodn. 46, 1424 (2012) (in Russian).

3. D. Barate, R. Teissier, Y. Wang, A.N. Baranov. Short wavelength intersubband emission from InAs/AlSb quantum cascade structures. Appl. Phys. Lett. 87, 051103 (2005) [DOI: 10.1063/1.2007854].

4. M.J. Yang, P.J. Lin-Chung, R.J. Wagner, J.R. Waterman, W.J. Moore, B.V. Shanabrook. Far-infrared spectroscopy in strained AlSb/InAs/AlSb quantum wells. Semicond. Sci. Technol. 8, S129 (1993) [DOI: 10.1088/02681242/8/1S/029].

5. M.J. Yang, P.J. Lin-Chung, B.V. Shanabrook, J.R. Waterman, R.J. Wagner, W.J. Moore. Enhancement of cyclotron mass in semiconductor quantum wells. Phys. Rev. B 47 , 1691 (1993) [DOI: 10.1103/PhysRevB.47.1691].

6. C. Gauer, J. Scriba, A. Wixforth, J.P. Kotthaus, C.R. Bolognesi, C. Nguyen, B. Brar, H. Kroemer. Energy-dependent cyclotron mass in InAs/AlSb quantum wells. Semicond. Sci. Technol. 9, 1580 (1994) [DOI: 10.1088/02681242/9/9/002].

7. R.J. Warburton, C. Gauer, A. Wixforth, J.P. Kotthaus, B. Brar, H. Kroemer. Intersubband resonances in InAs / AlSb quantum wells: Selection rules, matrix elements, and the depolarization field. Phys. Rev. B 53, 7903 (1996) [DOI: 10.1103/PhysRevB.53.7903].

8. V.Ya. Aleshkin, V.I. Gavrilenko, A.V. Ikonnikov, Yu.G. Sadofyev, J.P.Bird, S.R. Jonhson, Y.-H. Zhang. A cyclotrone resonance in both doped and non-doped InAs/AlSb heterostructures with quantum wells. Fiz. Tekh. Poluprovodn. 39, 71 (2005) (in Russian).

9. I.M. Tsydilkovskii. Electrons and Holes in Semiconductors (Nauka, 1972) (in Russian).

10. B.M. Askerov, Electron Transport Phenomena in Semiconductors (World Scientific, 1994) [ISBN: 978-981-021283-4].

Received 16.03.16 Translated from Russian by O.I. Voitenko

П.Ж. Байматов, Б.Т. Абдулазізов

КОНЦЕНТРАЦІЙНА ЗАЛЕЖНІСТЬ ЕФЕКТИВНОЇ МАСИ ЕЛЕКТРОНА, ЕНЕРГІЇ ФЕРМІ І ЗАПОВНЕННЯ МІНІЗОН У ЛЕГОВАНІЙ KВАНТОВІЙ ЯМI InAs/AlSb

$\mathrm{P}$ е $з$ ю м е

Представлено результати дослідження властивостей виродженого двовимірного електронного газу в одиночній гетероструктурній квантовій ямі InAs/AlSb. Непараболічності зони провідності InAs i AlSb описуються простою моделлю Кейна. Обчислено дисперсійні криві для перших трьох мінізон. Представлено також результати розрахунків енергії Фермі, концентрації в мінізонах і ефективної маси електронів на рівні Фермі як функції повної двовимірної концентрації. Отримані результати задовільно узгоджуються 3 експериментальними даними. 